IUTAM Symposium on Fluid-Structure Interaction in Ocean Engineering

June 2008 ; Volume 8 : Pages 57-68

http://dx.doi.org/10.1007/978-1-4020-8630-4

(c) 2008 Springer. Part of Springer Science+Business

Media

The original publication is available at http://www.springerlink.com
Archimer, archive institutionnelle de l'Ifremer http://www.ifremer.fr/docelec/

\title{
Experimental and numerical results on VIV and WIO
}

\author{
Benoît Gaurier ${ }^{1,{ }^{*}}$, Grégory Germain ${ }^{1}$, Marc Le Boulluec $^{2}$, Eric Giry ${ }^{3}$ and Emmanuel Fontaine ${ }^{4}$
}

\author{
${ }^{1}$ Ifremer, Hydrodynamics and Metocean, 62321 Boulogne-sur-Mer, France \\ 2 Ifremer, Hydrodynamics and Metocean, 29270 Plouzané, France \\ ${ }^{3}$ Saipem s.a., av. San Fernando, 78884 St Quentin en Yvelines, France \\ ${ }^{4}$ Ifp, av. Bois Préau, 92852 Rueil Malmaison, France \\ *: Corresponding author : B. Gaurier, email address : benoit.gaurier@ifremer.fr
}

\begin{abstract}
:
Vortex-Induced Vibrations (VIV) are well-known and related to the majority of cylindrical structures subjected to strong winds or currents. The VIV limit the lifetime of the structure because they increase the forces and so the fatigue. When several structures of this kind are put together in close interaction, the wake effects (Wake Induced Oscillations - WIO) sometimes involve strong instabilities. If these structures are flexible or mobile, oscillations of several diameters can be observed and collisions can occur ([4] \& [6]). Such structures are widespread in the oil industry where the extraction of oil in deep water can be done by means of risers. In some cases, risers are connected to a floating support called FPSO (Floating Production Storage Offloading) and held in tension by buoys (figure 2). These buoys are located at depth where waves do not have any significant influence. However, in these areas, the magnitude of currents can be sometimes important. Consequently, engineering companies have to find solutions to prevent hydrodynamic interactions between risers and buoys. For a better understanding and characterization of wake effects, an experimental study is carried out at the Ifremer (Institut Fran, cais de Recherche pour l'Exploitation de la Mer) flume tank in Boulogne-sur-Mer, France. This work is completed within the framework of the project Clarom cepm co 3007/04, in partnership with Doris engineering, Saipem s.a., Institut Fran, cais du P'etrole, Oceanide, Ecole Centrale Marseille \& Total. After presenting the experimental setup, we will focus on the interaction effects between two cylinders in close proximity. In this study, we quantify wake effects and risks of collision between structures. Several orientations and spacings between the cylinders are considered and tested. A short numerical study performed with the CFD code Fluent is also presented, for which hydrodynamic coefficients and motions of a single cylinder in a flow are numerically evaluated.
\end{abstract}




\section{Experimental setup}

The experimental study takes place at the IFREMER free surface flume tank located in Boulogne-sur-Mer, France (Figure 1). The flume tank is $18 \mathrm{~m}$ long by $4 \mathrm{~m}$ wide and $2 \mathrm{~m}$ deep with a side observation window of $8 \mathrm{~m} \times 2 \mathrm{~m}$. The flow turbulence is less than $5 \%$ and the flow velocity range is 0.1 to $2.2 \mathrm{~m} / \mathrm{s}$. Displacements of the models are measured with a $2 \mathrm{D}$ video system at a frequency of $25 \mathrm{~Hz}$.
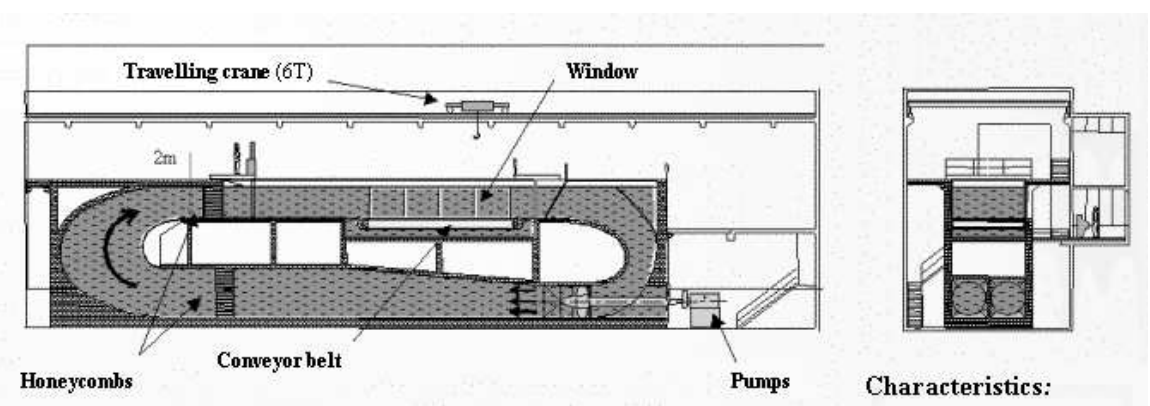

Fig. 1. Presentation of the Ifremer flume tank

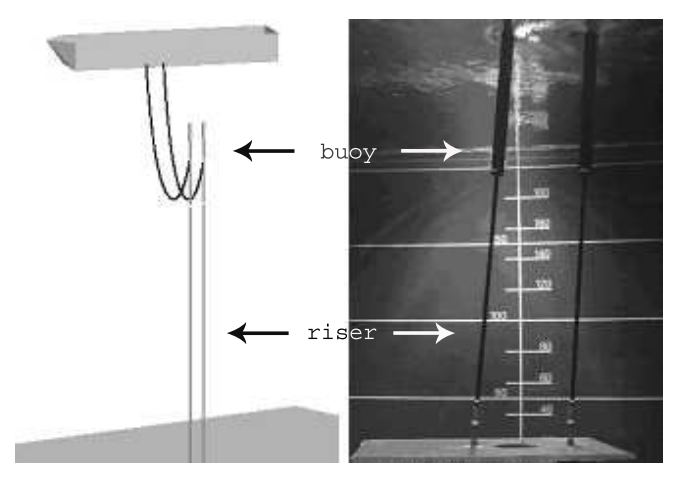

Fig. 2. Studied device and experimental setup

The experimental setup is shown on figures $2 \& 3$. It is derived from a real case provided by engineering. The studied configuration is an unfavourable case, i.e. buoys are intentionally placed at the same depth (figure 2) to observe strong hydrodynamic phenomena of interaction. Each cylinder reproduces a buoy (foam rod) and a part of riser (carbon rod) (figure 3). All the motion results presented here are given in the middle of the foam cylinder. The characteristics of the experimental setup result from the mathematical 
model suggested in [11], considering a pendular motion of a cylinder elastically mounted. Dynamics is governed by the pendulum equation for the angular time-dependent displacement $\theta(t)$ :

$$
I \ddot{\theta}_{i}(t)+B \dot{\theta}_{i}(t)+K \theta_{i}(t)=M_{i}(t)
$$

$M_{i}(t)$ indicates the moments due to the fluid forces applied on the cylinder, $I$ is the moment of inertia of the device in rotation, $B$ the structural damping and $K$ the angular stiffness. The subscript $i$ indicates the direction of the motion. The angles are always supposed to be small and the height of the cylinder $L$ is supposed to be large compared to the diameter $D$.

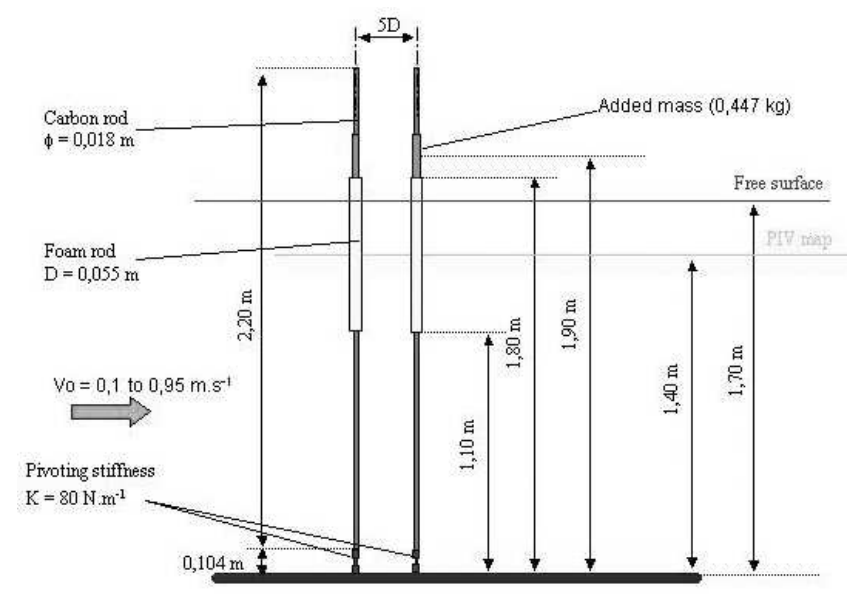

Fig. 3. Experimental setup for two closely spaced cylinders in tandem.

Parameters are selected to represent the real device with respect to the constraints related to the instrumentation. The damping coefficient $\zeta$ defined as $B /\left(2 \sqrt{K\left(I+I_{a}\right)}\right)$ is fixed at an average value of $50 \%$, with $I_{a}$ the added moment of inertia (the added mass coefficient $C_{m}$ is here estimated to one). The reduced mass $m^{*}$ defined as $I / I_{a}$ is not preserved here due to experimental considerations. Indeed $m^{*}$ is equal to 1.77 instead of 0.8 for the real case. The reduced velocity $V_{r}$ equal to $U /\left(f_{n} D\right)$, with $f_{n}$ the natural frequency of the model and $U$ the far upstream velocity of the current, is conserved.

For the wake effects study, in order to quantify the risks of collision, five initial configurations are considered (figure 4): case $1 \& 2$ : in-line configurations with initial spacing of $5 D$ and $7 D$, case $3 \& 4$ : shifted configurations of an angle of $11.5^{\circ}$ at $5 D \& 7 D$ and case 5 : shifted configurations of an angle of $90^{\circ}$ at $5 \mathrm{D}$. The influence of the relative positioning of the cylinders is studied for these five cases. 


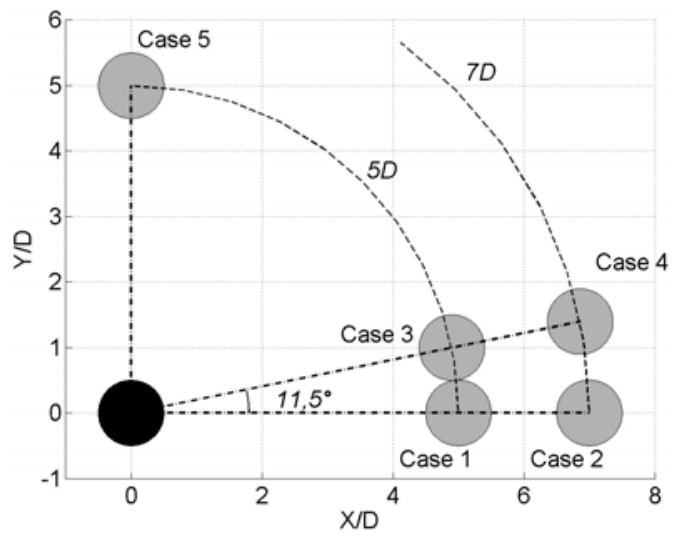

Fig. 4. Initial configurations of the trials carried out. The black circle represents the upstream cylinder. The grey circle stands for the downstream cylinder which occupies various initial positions. Current comes from the left.

\section{Wake effect study}

In order to observe interactions due to the current between the two cylinders, on a wide range of reduced velocity $\left(3<V_{r}<24\right)$, we perform trials for a velocity between 0.1 to $0.95 \mathrm{~m} / \mathrm{s}$ by step of $0.02 \mathrm{~m} / \mathrm{s}$ at the lock-in and 0.05 $\mathrm{m} / \mathrm{s}$ around. It represents 25 measurements for each of the 5 cases presented on figure 4. The corresponding Reynolds number varies from 25500 to 52000 .

A preliminary study is undertaken on a single cylinder [5] (results not shown here). This work gives us a better understanding of the behaviour of a single cylinder, to characterize the wake effects in the case of two cylinders. In addition, an interesting database is so established to conduct numerical comparisons and validations of CFD codes on a standard case.

In the case of two cylinders in tandem arrangement with an initial spacing of $5 D$ (case 1, figure 4), the behaviour of the upstream riser (figure 5) is not modified by the presence of the downstream riser in its wake. Amplitudes of transverse and in-line motions of this upstream cylinder (figure 6) are indeed very close to those observed for a single cylinder at same reduced velocities. Considering the low reduced mass of the device, the lock-in phenomena is noticed for a broader range of reduced velocity, between 5 and 10 compared to the results generally obtained ([3], [7] \& [14]). The figure 6 (c) shows the maximum minus the average value of each displacement. The amplification of the displacements of the upstream cylinder due to VIV reach the maximum value of $1.4 D$ at $V_{r}$ equal to 9 . The same behaviour is also observed for a single cylinder. For the case 1 and for $V_{r}<15$, the shielding effects or VIV modifications due to the presence of the downstream cylinder don't modify the behaviour of the upstream cylinder. 

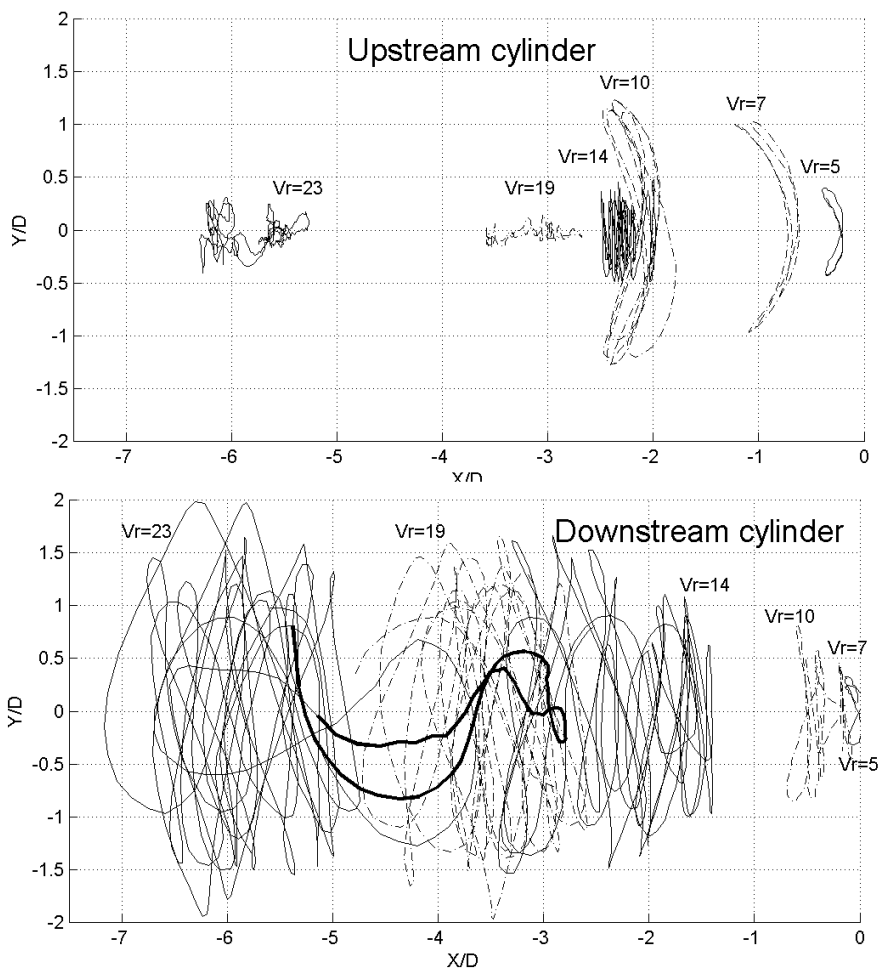

Fig. 5. Trajectories of the upstream and downstream risers at different reduced velocities, tandem arrangement at $5 D$ (case 1, figure 4). Current comes from the right.

For the downstream cylinder, wake effects due to the upstream cylinder influence largely its behaviour. Indeed, the reduction of the average velocity in the wake of the upstream cylinder modifies the hydrodynamic forces on the downstream cylinder ([16], [15] and [2]). There is a significant reduction of the amplitudes of oscillations and of the moving back of the downstream cylinder for $V_{r} \leq 10$. For higher reduced velocities $\left(V_{r}>10\right)$ the amplitude response of the downstream cylinder is chaotic. It can reach sometimes values upper or equal to two diameters for $V_{r}>15$ (figure 6 (c)). For these high reduced velocities, it can not be VIV effects; displacements are amplified by wake effects.

However, for $5<V_{r}<14$, VIV effects can be observed. Indeed, the standard deviation of the transverse response of the downstream cylinder (figure 6 (b)) shows two maximums, described by [12] and [9]. The first one of $0.59 D$ at $V_{r}$ equal to 8.5 corresponds to the lock-in of the first cylinder. The second one with a larger amplitude of $0.65 D$ at $V_{r}$ equal to 11 is also observable on the in-line response. This is a kind of lock-in for the downstream cylinder 

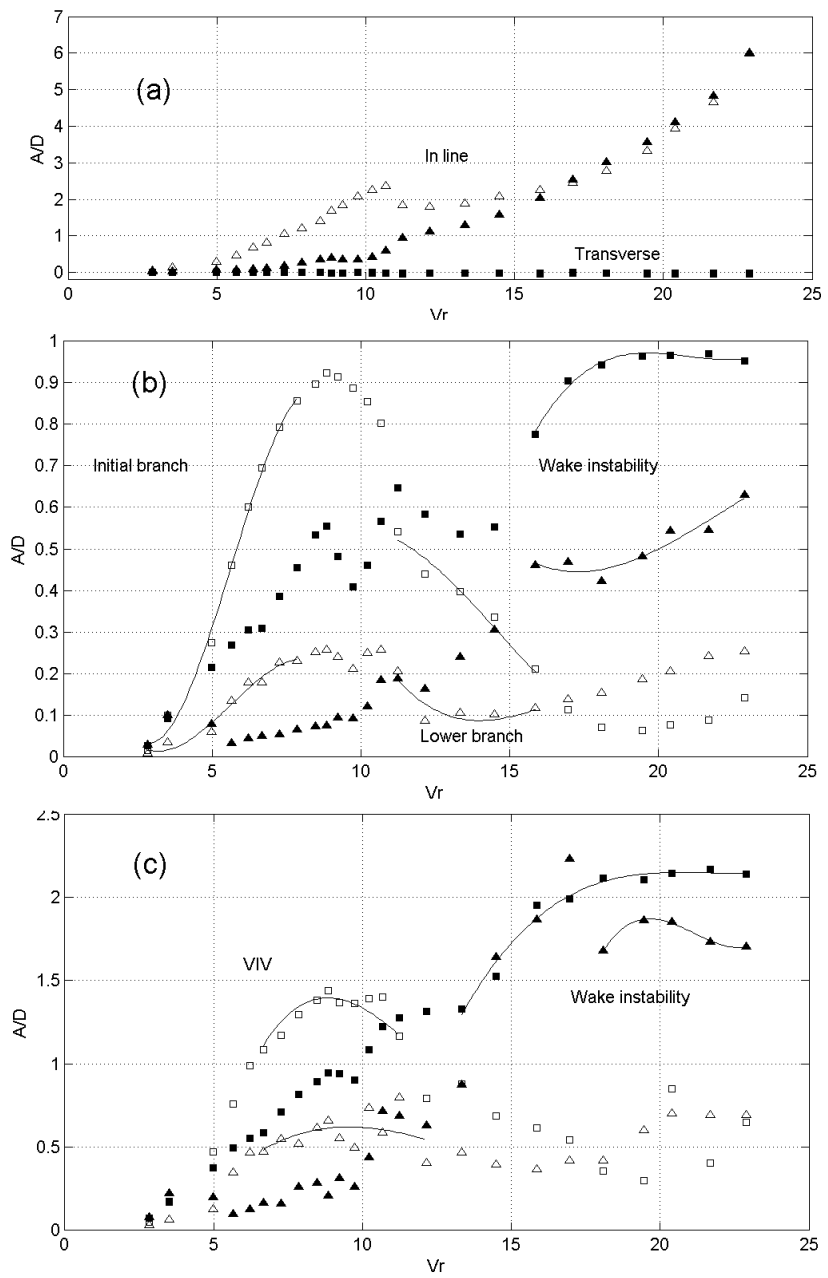

Fig. 6. Response of each cylinders expressed by reduced amplitude $A / D$ versus reduced velocity $V_{r}$ for the case 1 (tandem arrangement at $5 D$ ). $\square$ and $\triangle$ : transverse and in-line oscillations of upstream cylinder, $\mathbf{\square}$ and $\boldsymbol{\Delta}$ : transverse and in-line oscillations of downstream cylinder. (a): mean, (b): standard deviation, (c): maximum minus mean.

which appears at higher reduced velocities due to the reduction of the average velocity in the wake of the upstream cylinder [8].

The observed wake effects can be exceptionally intense as shown on figure 5 for $V_{r}=23$ (bold curve). Wake induced oscillations observed here, can reach some critical motions, which can generate collisions between risers.

As describe by [12] collisions are a brief phenomenon. They appear here at relatively low reduced velocities ( $V_{r}$ about 19$)$, contrary to previous results 
presented in [16]. For the initial spacing of $7 D$ (case 2, figure 4), results are quite similar. However, for the highest velocities, particular motions of the upstream cylinder occurs especially for the in-line oscillations, which can reach sometimes $2 D$.
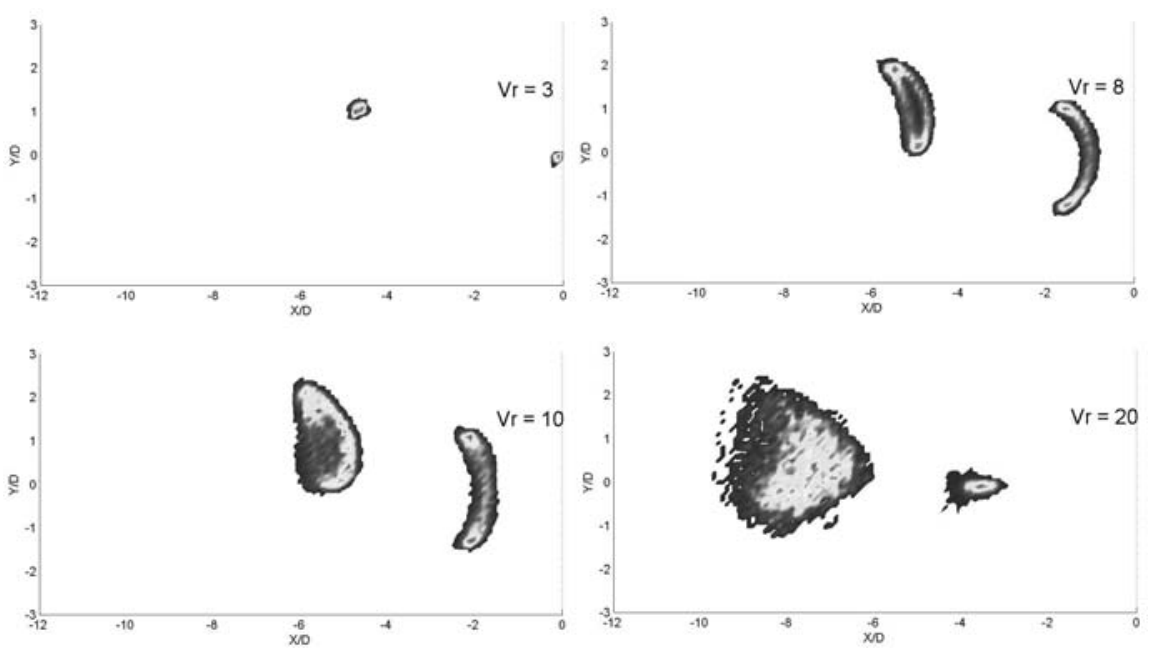

Fig. 7. Trajectories of the cylinders (for case 3) for different $V_{r}$. The gray scale indicates the presence rate of the cylinders.

Results obtained for the shifted configurations are quite similar to the in-line arrangements. However, the downstream cylinder tends to come back in the wake of the upstream one, when the reduced velocity increases. A dissymmetry is observed in the behaviour of the downstream cylinder and trajectories are not identical to those previously shown (figure 7). So, the cylinders are often in close proximity and many collisions occur when $V_{r}$ is upper to 19. Between shifted arrangement at $5 D$ and $7 D$ (cases 3 and 4 , figure 4) a difference for the higher reduced velocities is remarked: the more the cylinders are far, the less the amplitude of oscillations of the downstream cylinder is large [1]. In fact, at $5 D$ and $V_{r}$ equal to 20 the transverse region is around $4 D$ large whereas at $7 D$ and for the same reduced velocity the transverse region is only $2.5 \mathrm{D}$ large.

For the case 5 (figure 4), the two cylinders behave like an isolated one. Transverse oscillations are just a few dissymmetrical. In fact the cylinders tend to deviate and move away from a value remaining lower than $0.5 D$. Contrary to the previous cases, no collision occurs during these trials. 


\section{Collisions study}

When the cylinders are in close proximity like described previously, collisions can occur at the top of the structures. These phenomena are observed when the reduced velocity is upper or equal to 19 for all the studied cases (except the case 5). The analysis of the behaviour of each cylinders versus time shows two types of collision. Type 1: the downstream cylinder is sucked by the upstream cylinder. Type 2: the upstream cylinder is suddenly animated by large oscillations and moves back.
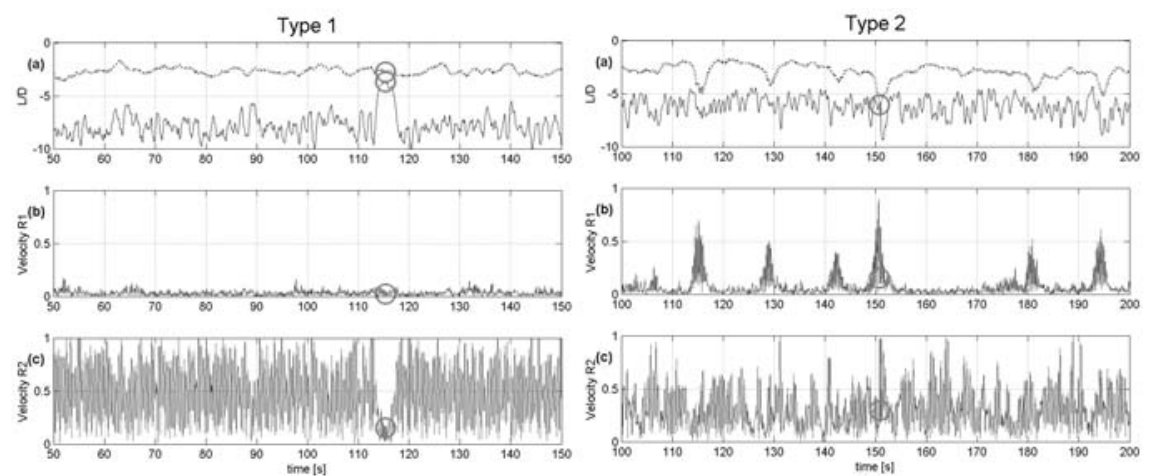

Fig. 8. Two types of collision at $V_{r}=23$ (on the left: case 1, on the right: case 4 from the figure 4). (a): reduced distance from the initial point for each cylinders. Dot line: upstream cylinder, solid line: downstream cylinder. (b) \& (c): instantaneous velocity of respectively upstream and downstream cylinders (in $\mathrm{m} / \mathrm{s}$ ).

The instantaneous velocity of the downstream cylinder during the type 1 collision (figure 8) decreases strongly and briefly. The downstream cylinder is slowly sucked. The upstream cylinder is not disturbed by the change of behaviour of the downstream one.

Contrary to the type 1 collision, the upstream cylinder is moving back during the type 2 collision. The instantaneous velocity of the upstream cylinder (figure 8) shows many peaks. For each peak of the velocity, the upstream cylinder moves back and the distance between the two cylinders decreases. This sudden change of behaviour of the upstream cylinder is much more frequent than for collision of type 1 , but do not always lead to a collision between cylinders.

The study of collisions recorded during the trials shows that the appearance frequency of collisions is increasing with velocity. However, at a given velocity, this frequency differs between the different tested cases (figure 9). Type 1 collisions are only observed for initial spacing of $5 D$ between cylinders (cases 1 \& 3). In addition, the two type of collisions are together noticed for the only shifted configuration at $5 D$ (case 3 ). 

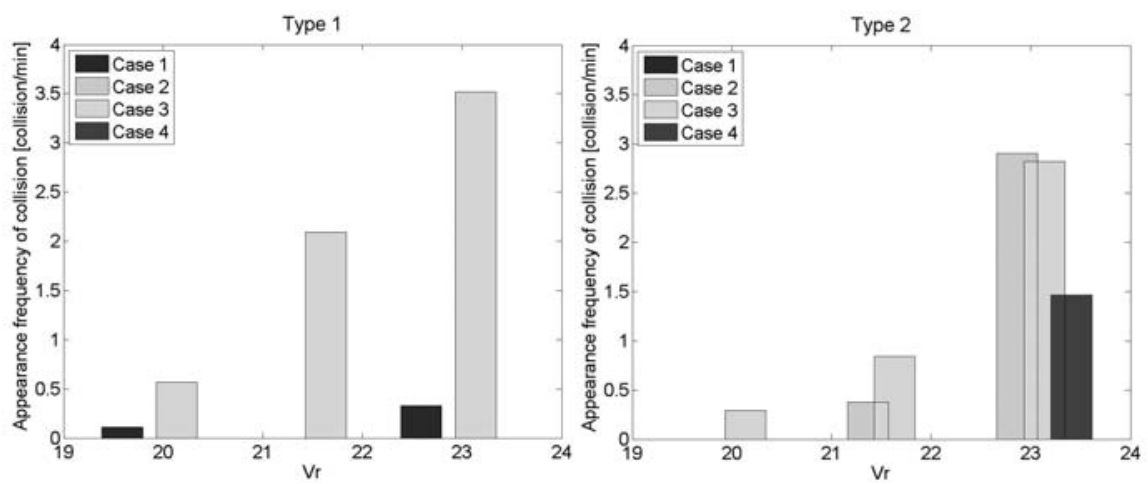

Fig. 9. Appearance frequency of type 1 and type 2 collisions for all the tested cases versus the reduced velocity.

\section{Numerical study}

Numerical simulations are performed with the CFD code Fluent. A twodimensional model of cylinder is implemented. The flow is solved in two dimensions on a plane perpendicular to the cylinder axis. It is first animated with forced transverse sinusoidal oscillations $y(t)=A \cos \left(\omega t+\varphi_{y}\right)$, in a current, in order to check the capacity of the code to calculate accurately hydrodynamic coefficients such as added mass or drag.

The transverse force computed by Fluent is supposed to be sinusoidal: $F_{y}=F_{m} \cos \left(\omega t+\varphi_{F}\right)$. This force can be compared to the "Morison" equation. According to this equation, the time-dependent force exerted on a body moving with the velocity $\dot{y}(t)$ in a fluid is assumed to be a linear sum of an acceleration-dependent inertial force (with $C_{m}$ the added mass coefficient) and a velocity-square-dependent drag force (with $C_{d}$ the drag coefficient):

$$
F(t)=-\rho C_{m} L \frac{\pi D^{2}}{4} \ddot{y}(t)-\frac{1}{2} \rho C_{d} D L \dot{y}(t)|\dot{y}(t)|
$$

According to [14] the drag term of equation 2 can be linearized to finally obtain (with $\varphi=\varphi_{F}-\varphi_{y}$ ):

$$
C_{m}=\frac{F_{m} \cos \varphi}{\rho L \frac{\pi D^{2}}{4} A \omega^{2}} \quad \text { and } \quad C_{d}=\frac{F_{m} \sin \varphi}{\frac{8}{6 \pi} \rho D L A^{2} \omega^{2}}
$$

The amplitude of the transverse force $F_{m}$ is determined by calculating the Fourier component at the motion frequency of the transverse force given by Fluent.

The results (figure 10) reveal that $C_{m}$ decreases sharply from about 2 to about -0.5 as the reduced velocity increases from $V_{r} \simeq 4.5$ to 5.2 . For low reduced velocity, the added mass coefficient is close to one. This phenomenon occurs for the three Reynolds numbers tested here, but it is stronger for the 

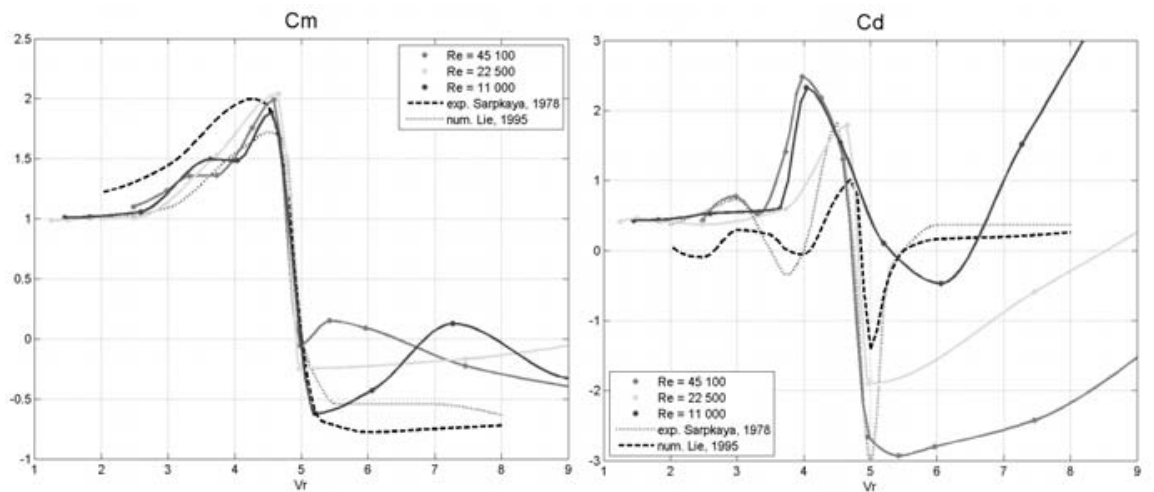

Fig. 10. Added mass coefficient $C_{m}$ and drag coefficient $C_{d}$ versus reduced velocity for 3 different Reynolds numbers compared with [13] and [10].

lower Reynolds number. The decrease of $C_{m}$ from a large positive value toward zero as $V_{r}$ approaches 5 shows that the oscillation frequency is rising toward the vortex shielding frequency.

Figure 10 also shows that the drag coefficient $C_{d}$ rises sharply from about 0.4 to 2.2 for $V_{r} \leq 4.5$. The drag is in phase with the direction of motion of the cylinder and helps to magnify the oscillations.

Generally these results are in quite good agreement with the bibliographical results for $V_{r} \leq 5$. The drag coefficient shows however a larger deviation.

2D and 3D simulations with free oscillations are also performed to investigate the dynamic response of a single cylinder. The stiffness of the equivalent model is adjusted so that the natural frequency corresponds to the experimental model. Figure 11 shows the resulting trajectories of the centre of the cylinder section for a reduced velocity of 5 .

Numerical results are in quite good agreement with the experiments until $V_{r}$ about 10. In-line and transverse amplitudes of oscillations are comparable to the experimental results in spite of the difference of imposed motions. Numerical simulations take into account a motion of translation while the experimental cylinder is in rotation. For higher velocity, the 3D simulations are much more relevant than the $2 \mathrm{D}$ ones. This is due to the correlation length along the cylinder, i.e. the spanwise synchronization length of vortex, considered for the $3 \mathrm{D}$ simulations.

\section{Conclusion}

The study of interaction between two rigid cylinders elastically mounted and submitted to current shows that the response of the downstream cylinder is 

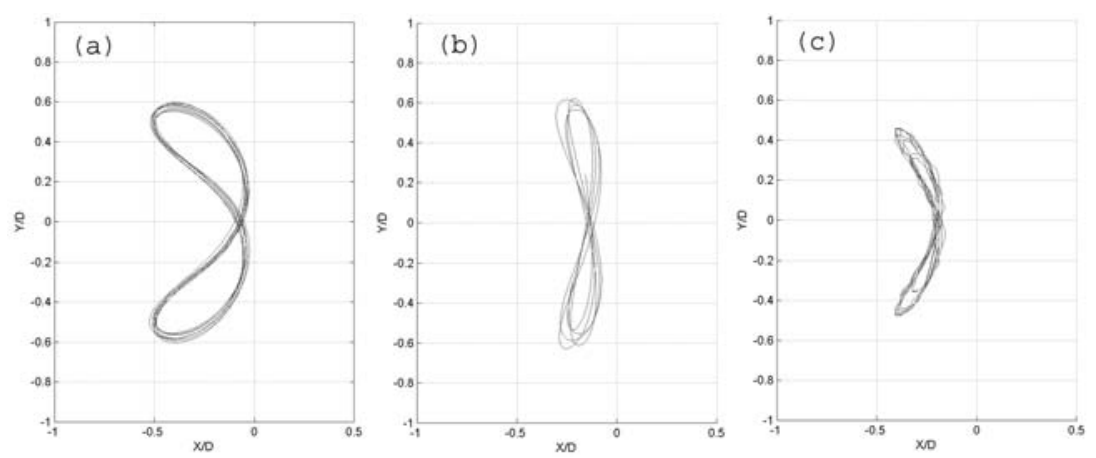

Fig. 11. Trajectories of the centre of the cylinder at $V_{r}=5$. (a): 2-dimensional simulation, (b): 3-dimensional simulation and (c): experimental result. Current comes from the right.

very different from a single one. Except when the cylinders are shifted of an angle of $90^{\circ}$, the downstream cylinder is strongly affected by wake effects.

In spite of an increase of the oscillation amplitudes of the downstream cylinder, there is no real lock-in at the expected reduced velocity. However the fluctuations of the transverse motions are larger than those of the upstream cylinder for reduced velocity greater than 10 . In addition these motions increase after the lock-in of the upstream cylinder and they can sometimes reach more than 2 diameters for the highest velocity.

The upstream cylinder is not too much disturbed by the downstream cylinder in its wake. Its response is similar to a single cylinder for reduced velocity lower than 17. For higher velocity, sudden changes of behaviour appear which can cause collisions between cylinders.

The numerical study shows that first simulations obtained with the CFD code Fluent are in quite good agreement with bibliography results. Hydrodynamic coefficients like added mass coefficient and drag coefficient as well as the motions of the cylinder are correctly reproduced for the lowest reduced velocities. This numerical work is still in progress but it gives good hopes for the following studies.

\section{References}

1. G.R.S. Assi, J.R. Meneghini, J.A.P. Aranha, P.W. Bearman, and E. Casaprima. Experimental investigation of flow-induced vibrations interference between two cylinders in tandem arrangement. In OMAE, Halkidiki, GREECE, 2005.

2. R.D. Blevins. Model for forces and stability of a cylinder in a wake. In Flow Induced Vibration, Ecole Polytechnique, FRANCE, 2004.

3. C.C. Feng. The measurements of vortex-induced effects in flow past a stationary and oscillating circular cylinder. Master's thesis, University of British Columbia, Vancouver, CANADA, 1968. 
4. E. Fontaine, J. P. Morel, S. Blarel, Y. M. Scolan, D. Teissier, T. Rippol, F. Petrie, G. Deleuil, E. Giry, and P. Lespinasse. Ongoing research on risers hydrodynamic interference. In International Symposium on Technology of Ultra Deep Ocean Engineering, Tokyo, JAPAN, Feb.1-2 2005.

5. B. Gaurier, G. Germain, M. Le Boulluec, E. Giry, and E. Fontaine. Caracterisation d'effets de sillages rencontres en milieu offshore. In 11emes journees de l'hydrodynamique, Brest, FRANCE, Apr.3-5 2007.

6. G. Germain, B. Gaurier, M. Le Boulluec, E. Fontaine, and J. Capul. Vortex and wake effects on closely spaced marine risers. In Flow Induced Vibration PVP ASME, Vancouver, CANADA, Jul.23-27 2006.

7. R. Govardhan and C. H. K. Williamson. Modes of vortex formation and frequency response of a freely vibrating cylinder. Journal of Fluid Mechanics, 420:85-130, 2000.

8. E. Huse. Intercation in deep-sea riser array. In Offshore technology conference, OTC 7237, 1993.

9. A. Laneville and D. Brika. The fluid and mechanical coupling between two circular cylinders in tandem arrangement. Journal of Fluids and Structures, 13:967-986, 1999.

10. H. Lie. A time domain model for simulation of vortex-induced vibrations on a cable. In Flow Induced Vibration, pages 455-462, Honolulu, HAWAII, Jul.23-27, 1995.

11. J.P. Morel, S. Blarel, G. Germain, and J.V. Facq. Risers in tandem: wake induced oscillations modeling for basin testing. Technical report, Ifremer, 2005.

12. S.I. Sagatun, K. Herfjord, and T. Holmas. Dynamic simulation of marine risers moving relative to each other due to vortex and wake effects. Journal of Fluids and Structures, 16(3):375-390, 2002.

13. T. Sarpkaya. Fluid forces on oscillating cylinders. Journal of Fluid Mechanics, 104:275-290, 1978.

14. T. Sarpkaya. A critical review of the intrinsic nature of vortex-induced vibrations. Journal of Fluids and Structures, 19:389-447, 2004.

15. H. Schlichting. Boundary-Layer Theory. McGrow-Hill Book Company, Reissued 1987.

16. W. Wu, S. Huang, and N. Barltrop. Multiple stable/unstable equilibria of a cylinder in the wake of an upstrema cylinder. In $O M A E$, Rio de Janeiro, BRAZIL, 2001. 


\section{Index}

Collision, 1, 3, 6-8, 11

Cylinder, 1-11

Experimental, 1, 2, 10

Fluent, 1, 9, 11

Numerical simulation, 1, 4, 9-11
Riser, 1, 4, 6

Tank, 1, 2

Vortex-Induced Vibrations, 1, 4, 5

Wake, 1, 3-6 